\title{
Tetra-tert-butyl-dihydropentafulvalen als Edukt für para- und diamagnetische Nickelkomplexe
}

\author{
Peter Jutzi *, Jörg Schnittger, Wolfram Wieland, Beate Neumann \\ und Hans-Georg Stammler
}

Fakultät für Chemie der Universität Bielefeld, Universitätsstraße, W-4800 Bielefeld I (Deutschland)

(Eingegangen den 17. April 1991)

\begin{abstract}
Reaction of 1,1',3,3'-tetra-tert-butyl-5,5'-dihydropentafulvalene (1) with methyllithium and subsequent treatment with an excess of tetracarbonylnickel leads to the isomeric, paramagnetic complexes $\left(\eta^{5}: \eta^{5}-{ }^{t} \mathrm{Bu}_{4} \mathrm{C}_{10} \mathrm{H}_{4}\right)\left(\eta^{2}: \eta^{2}{ }^{\mathrm{t}} \mathrm{Bu}_{4} \mathrm{C}_{10} \mathrm{H}_{6}(\mathrm{CO})_{2} \mathrm{Ni}_{2}\right.$ (2a) and $\left(\eta^{5}: \eta^{2}-{ }^{t} \mathrm{Bu}_{4} \mathrm{C}_{10} \mathrm{H}_{5}\right)_{2}(\mathrm{CO})_{2} \mathrm{Ni}_{2}$ (2b). Treatment of $2 \mathrm{a}$ with $\left.\mathrm{Br}_{2} / \mathrm{P}_{[} \mathrm{C}_{6} \mathrm{H}_{5}\right]_{3}$, and with $\left.\mathrm{I}_{2} / \mathrm{P}^{2} \mathrm{C}_{6} \mathrm{H}_{5}\right]_{3}$, respectively, affords by elimination of 1 the compounds $\left.\left(\eta^{5}: \eta^{5}-{ }^{t} \mathrm{Bu}_{4} \mathrm{C}_{10} \mathrm{H}_{4}\right)\left(\mathrm{P}_{2} \mathrm{C}_{6} \mathrm{H}_{3}\right]_{3}\right)_{2} \mathrm{Ni}_{2} \mathrm{X}_{2}$, with $\mathrm{X}=\mathrm{Br}(3)$ and with $\mathrm{X}=\mathrm{I}(4)$, respectively. By treatment of $\mathbf{2 b}$ with bromine the paramagnetic $\mathrm{Ni}^{\mathrm{III}}$-species $\left(\eta^{5}-{ }^{t} \mathrm{Bu}_{2} \mathrm{C}_{5} \mathrm{H}_{2}\right)\left({ }^{t} \mathrm{Bu}_{2} \mathrm{C}_{5} \mathrm{H}_{3}\right) \mathrm{NiBr}_{2}$ (5) is obtained. The solid state structure of 5 is discussed.
\end{abstract}

\section{Zusammenfassung}

Reaktion von 1,1',3,3'-Tetra-tert-butyl-5,5'-dihydropentafulvalen (1) mit Methyllithium und anschliessende Umsetzung mit überschüssigem Tetracarbonylnickel führt zum Isomerengemisch der paramagnetischen Komplexe $\left(\eta^{5}: \eta^{5}-{ }^{t} \mathrm{Bu}_{4} \mathrm{C}_{10} \mathrm{H}_{4}\right)\left(\eta^{2}: \eta^{5}-{ }^{t} \mathrm{Bu}_{4} \mathrm{C}_{10} \mathrm{H}_{6}(\mathrm{CO})_{2} \mathrm{Ni}_{2}\right.$ (2a) und $\left(\eta^{5}: \eta^{2}-{ }^{t} \mathrm{Bu}_{4}\right.$ $\left.\mathrm{C}_{10} \mathrm{H}_{5}\right)_{2}(\mathrm{CO})_{2} \mathrm{Ni}_{2}$ (2b). Umsetzung von $2 \mathrm{a}$ mit $\left.\mathrm{Br}_{2} / \mathrm{P}_{6} \mathrm{C}_{6} \mathrm{H}_{5}\right]_{3}$ bzw. mit $\left.\mathrm{I}_{2} / \mathrm{P} \mathrm{C}_{6} \mathrm{H}_{5}\right]_{3}$ liefert unter Freisetzung von 1 die Verbindungen $\left.\left(\eta^{5}: \eta^{5}-{ }^{t} \mathrm{Bu}_{4} \mathrm{C}_{10}-\mathrm{H}_{4}\right)\left(\mathrm{P}_{[} \mathrm{C}_{6} \mathrm{H}_{5}\right]_{3}\right)_{2} \mathrm{Ni}_{2} \mathrm{X}_{2}$ mit $\mathrm{X}=\mathrm{Br}(3)$ bzw. $\mathrm{X}=\mathrm{I}$ (4). Durch Umsetzung von $2 \mathrm{~b}$ mit Brom wird die paramagnetische $\mathrm{Ni}^{1 \mathrm{III}}$-Spezies $\left(\eta^{5}-{ }^{1} \mathrm{Bu}_{2} \mathrm{C}_{5} \mathrm{H}_{2}\right)\left({ }^{t} \mathrm{Bu}_{2} \mathrm{C}_{5}\right.$ $\left.\mathrm{H}_{3}\right) \mathrm{NiBr}_{2}$ (5) erhalten. Die Festkörperstruktur von 5 wird diskutiert.

\section{Einleitung}

Cyclopentadienyl(Cp)-Liganden zeichnen sich durch cine große Variationsbreite der Bindungsbeziehung zu Metallen aus und dokumentieren damit ihre Fähigkeit, sich den elektronischen Verhältnissen anzupassen. In der Nickel-Chemie werden neben dem häufig vorkommenden $\eta^{5}$-koordinierten $\mathrm{Cp}$-Liganden auch $\eta^{1}-\eta^{2}$ - und $\eta^{3}$-Haptizitäten gefunden [1,2]. Im Rahmen der experimentellen Arbeiten mit dem Ligandensystem 1,1'3,3'-Tetra-tert-butyl-5,5'-dihydropentafulvalen (1) [3-6] interessierte uns die Funktionsweise dieses Liganden in der Nickel-Chemie. Wir berichten im Folgenden über die Synthese und die Charakterisierung von paramagnetischen Nickel(I)- und Nickel(III)- sowie diamagnetischen Nickel(II)- 
2

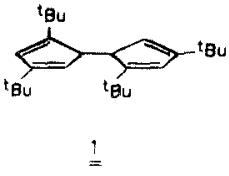

1) $2 \mathrm{MeLi}$ 2) $2 \mathrm{Ni}(\mathrm{CO})_{4}$

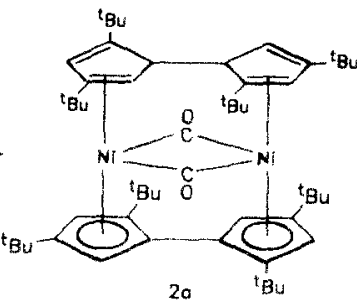

$\underline{20}$

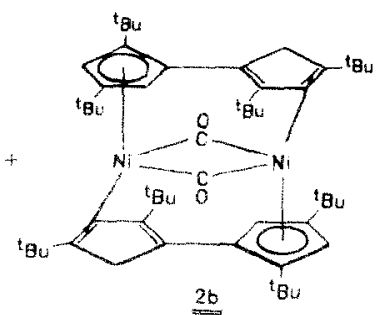

Schemal

Komplexen. Desweiteren beschreiben wir die Festkörperstruktur von Dibrom(1,3di-tert-butylcyclopentadienyl)-5.5'-(1'.3'-di-tert-butyl-2', $5^{\prime}$-cyclopentadien nickel (5). 5 ist ein weiteres Beispiel für einen paramagnetischen $\mathrm{Cp}$-Ni(III)-Komplex [7].

\section{Präparative Ergebnisse}

Gibt man zu einer THF-Lösung aus zwei Äquivalenten 1.1'.3.3'-Tetra-tert-butyl-

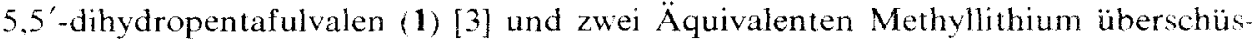
siges Tetracarbonylnickel, so wechselt die Farbe der Lösung augenblicklich von orange-gelb nach tief-braun. Erhitzt man sodann unter RückfluB, tritt nach ca. zwei Stunden ein Farbwechsel nach tiefrot ein. Durch chromatographische Aufarbeitung des Reaktionsgemisches an $\mathrm{Al}_{2} \mathrm{O}_{3}$ /Petrolether eluiert man eine kirschrote Fraktion. aus welcher nach Einengen ein rotes, röntgenamorphes Pulver ausfäll, welches aus Dicarbonyl(1.1',3.3'-tetra-tert-butyl-5,5'-pentafulvalen $)\left(1 . I^{\prime}, 3,3^{\prime}\right.$-tetra-tert-butyl5,5'-dihydropentafulvalen)dinickel (2a) und Dicarbonyl-bis(1,3-di-tert-butykyclopentadienyl)-5.5'-(1'.3'-di-tert-butylcyclopentadien)dinickel (2b) im Verhältnis 1: 5 besteht. Das Isomerengemisch aus $\mathbf{2 a / 2 b}$ ist gegenüber Luft ind Feuchtigkeit nahezu inert, wird aber von Mineralsäuren zersetzt. Kristalle konnten aufgrund der sehr guten Löslichkeit in allen gängigen organischen Solventien bislang nich erhalten werden.

Im ${ }^{1} \mathrm{H}$-NMR-Spektrum von $\mathbf{2 a} / \mathbf{2 b}$ treten im Bereich von $1.161 .39 \mathrm{ppm}$ ach Signale auf, die jeweils den tert-Butyl-Einheiten zugeordnet werden können. Je vier Signale davon stehen zueinander im Integralverhältnis $1: 1: 1: 1$; das Verhältnis der beiden Signalgruppen untereinander beträgt ca. 1:5. Fin breites Singulen erschein in dem für allylständige Ringprotonen typischen Bereich bei 3.10 ppm. Weitere Resonanzen werden im Bereich von 4.84-6.36 ppm beobachtet und stammen von vinylischen und aromatischen Protonen am Fulvalengerust. Line Temperaturabhängigkeit der 'H-NMR-Verschiebungen wird nicht festgestellt.

Im ${ }^{13} \mathrm{C}-\mathrm{NMR}$-Spektrum $\left(\mathrm{C}_{6} \mathrm{D}_{6}\right)$ von $\mathbf{2 a} / \mathbf{2 b}$ beobachtet man jeweils vier Resonanzen für die primären und die quartären Kohlenstoff-Atome der tert-Butylgruppen im Bereich von 30.68-34.20 ppm. Signale derjenigen Verbindungen. die in geringerer Ausbeute entstanden ist, sind weniger deutlich zu erkennen. $1 \mathrm{~m}$ Bereich von 91.35 und $157.01 \mathrm{ppm}$ treten Signalgruppen auf, welche vinylischen und aromatischen Ringkohlenstolf-Atomen zuzuordnen sind. Das Infrarot-Spektrum des Isomeren-Gemisches zeigt im carbonyl-relevanten Bereich eine Absorptionsbande bei $1831 \mathrm{~cm}^{-1}$ : anhand ihrer Lage ist auf verbrückende CO-Einheiten an shließen. 
Massenspektrometrische Untersuchungen bestätigen die Summenformel $\mathrm{C}_{52} \mathrm{H}_{82}$ $\mathrm{Ni}_{2}(\mathrm{CO})_{2}$. Das Molekül-Ion erscheint bei $m / z=878$. Man beobachtet den Verlust von Carbonyl-Einheiten und von tert-Butyl-Gruppen. Zudem treten Ionen mit $m / z=439$ und $m / z=411$ auf, letztere liefern den Basispeak des Spektrums. Diese beiden Signale sind den Fragmenten ${ }^{\mathrm{t}} \mathrm{Bu}_{4} \mathrm{C}_{10} \mathrm{H}_{5} \mathrm{NiCO}^{+}$und ${ }^{\mathrm{t}} \mathrm{Bu}_{4} \mathrm{C}_{10} \mathrm{H}_{5} \mathrm{Ni}^{+}$ zuzuordnen.

Die Bestimmung der molaren Suszeptibilität zeigt den Paramagnetismus von 2a/2b an. Aus dem Wert von $X_{\mathrm{Mol}}^{298 \mathrm{~K}}=+2851 \times 10^{-6} \mathrm{~cm}^{3} / \mathrm{Mol}$ errechnet sich ein magnetisches Moment von $2.58 \mu \mathrm{B}$, so daß auf das Vorhandensein von zwei ungepaarten Elektronen im Molekül geschlossenen werden muß. Eine MetallMetall-Bindung zwischen zwei Nickel-Zentren, wie im literaturbekannten Dicarbonyl(cyclopentadienyl)nickel-Dimeren beobachtet [8-12], liegt somit nicht vor. Formal befindet sich jedes Nickel-Atom in der Oxidationsstufe +1 .

Die für $\mathbf{2 a}$ und $\mathbf{2 b}$ vorgeschlagenen Strukturen sind im Reaktionsschema 1 abgebildet. Aus den spektroskopischen Daten geht hervor, daß in beiden Isomeren jeweils zwei Nickel-Atome symmetrisch durch zwei Carbonyl-Einheiten verbrückt sind. In der Koordinationssphäre der Metallzentren befinden sich je zwei ${ }^{\mathrm{t}} \mathrm{Bu}_{4} \mathrm{C}_{10^{-}}$ Systeme. Die Nickel-Atome sind sowohl $\eta^{2}$ - als auch $\eta^{5}$-artig an das Ligandensystem gebunden. In Verbindung 2a liegt ein Dihydrofulvalen-Ligand zweifach deprotoniert und $\eta^{5}: \eta^{5}$-gebunden vor, während der zweite als Dien $\eta^{2}: \eta^{2}$-koordiniert ist. Im Isomer $\mathbf{2 b}$ sind beide Dihydrofulvalen-Liganden einfach deprotoniert und $\eta^{5}: \eta^{2}$-koordiniert $\left[13^{*}\right]$. Eine definitive Zuordnung der Signale zu 2a oder 2b ist anhand der spektroskopischen Daten jedoch nicht möglich. Die getroffene Zuordnung resultiert vielmehr aus den zweifelsfrei aufgeklärten Folgereaktionen und den Ausbeuten an Reaktionsprodukten, die dem vorgegebenen Substratverhältnis $(\mathbf{2 a} / \mathbf{2} \mathbf{b}=1 / 5)$ entsprechen. Auch bleibt unklar, welches der beiden Doppelbindungssysteme in den Cyclopentadien-Einheiten von $\mathbf{2 a} / \mathbf{2 b}$ jeweils mit dem NickelAtom wechselwirkt; ebenfalls denkbare $\eta^{4}$-Bindungsbeziehungen der Cyclopentadien-Einheiten zum Nickel sollten aus elektronischen Gründen auszuschließen sein.

Die mit dem Isomerengemisch $\mathbf{2 a} / \mathbf{2 b}$ durchgeführten Reaktionen werden im Folgenden beschrieben. Bekannten Reaktionsprinzipien in der Nickelchemie folgend $[9,14]$, wird eine auf $-40^{\circ} \mathrm{C}$ gekühlte Diethylether-Lösung des Isomerengemisches 2a/2b zunächst mit einer Lösung von Brom in Diethylether und anschließend mit einer Lösung von Triphenylphosphan in Toluol versetzt. Das Reaktionsgemisch hellt sich beim Erwärmen auf Raumtemperatur auf. Nach Aufarbeitung isoliert man Dibrom-bis[triphenylphosphan] (1,1',3,3'-tetra-tert-butyl-5,5'-pentafulvalen)dinickel (3) in ca. 9 proz. Ausbeute in Form von dunkelroten Kristallen. Als weiteres Hauptprodukt wird NMR-spektroskopisch 1 nachgewiesen. Analog hierzu verläuft die Reaktion mit elementarem Iod und Triphenylphosphan. Als Produkt wird Diiod-bis[triphenylphosphan] (1,1',3,3'-tetra-tert-butyl-5,5'-pentafulvalen)dinickel (4) in gut 14 proz. Ausbeute als rote Kuben erhalten.

Die Verbindungen 3 und 4 sind gegenüber Luft und Feuchtigkeit beständig. Im ${ }^{1}$ H-NMR-Spektrum beider Spezies treten je vier Signale im Verhältnis 1:1:1:1 für die tert-Butylgruppen sowie jeweils vier Multipletts für die Protonen des Fulvalengerüstes auf. Die Protonen des Phenylkerns erscheinen als Multiplett im Bereich

\footnotetext{
* Die Literaturnummer mit einem Sternchen deutet eine Bemerkung in der Literaturliste an.
} 


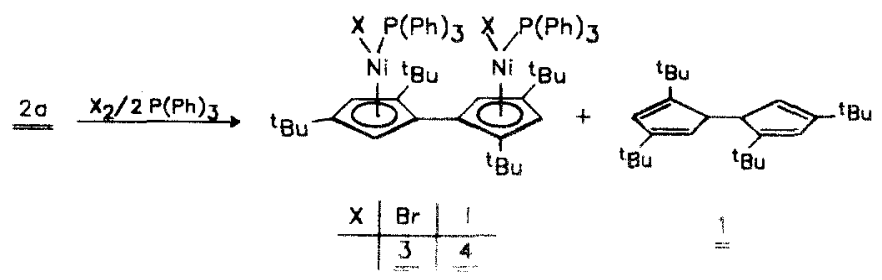

Schema 2.

von 7.03 bis $7.30 \mathrm{ppm}$. Im ${ }^{13} \mathrm{C}$-NMR-Spektrum der Verbindung 4 beobachtet man jeweils vier Signale für die primären und die quartären Kohlenstoffatome der tert-Butylgruppen. Es treten vier Resonanzen für die am Fulvalengerüst lokalisierten protonentragenden Kohlenstoffatome und sechs Signale fü die restlichen C-Atome auf.

Diese komplexen Spektren lassen sich damit erklären, daß3 die magnetische $\ddot{A} q u i v a l e n z$ von ${ }^{1} \mathrm{H}$ - und ${ }^{13} \mathrm{C}$-Kernen aufgehoben wird. Dies gescheht dadurch. daB die Triphenylphosphan-Liganden die Rotation um die zentrale Vi-Cp-Achs: einschränken. Sind in bisher bekannten Komplexen mit dem Tetra-tert-butyl-pentafulvalen-Liganden jeweils zwei der insgesamt vier tert-Butylgruppen chemisch äquivalent $[4-6]$, befinden sich in 3 und 4 alle tert-Butylgruppen $n$ bezug au ihre Uimgebung in unterschiedlichen Positionen.

Die Lagen der Phosphor-Resonanzen von $28.40 \mathrm{ppm}$ (3) und $36.80 \mathrm{ppm}$ (4) sind typisch für komplexgebundenes Phosphan. In den Massenspektren beider Verbin. dungen wird das Auftreten won Molekül-Ionen nicht beobachtet. Die Peaks mil den höchsten Massen sind Fragment-Ionen der Zusammensetzung ' $\mathrm{Bu}, \mathrm{C}_{10} \mathrm{H}_{5} \mathrm{NiBr}$ ' $\left(m / z=490\right.$, bezogen auf ${ }^{58} \mathrm{Ni}$ und $\left.{ }^{79} \mathrm{Br}\right)$ und ${ }^{1} \mathrm{Bu}_{4} \mathrm{C}_{\mathrm{i})} \mathrm{H}_{5} \mathrm{Ni}$ ( $m / 2=538$, bezogen auf ${ }^{5 x} \mathrm{Ni}$ und ${ }^{127}$ I) zuzuordnen. Durch Halogenverlust entsteht in heiden Fallen das Fragment-Ion ${ }^{1} \mathrm{Bu}_{4} \mathrm{C}_{10} \mathrm{H}_{5} \mathrm{Ni} \quad(m / z=411)$. Dic Signale mit den hochsten Intensitäten stammen von den Fragment-lonen $\mathrm{P}\left(\mathrm{C}_{6} \mathrm{H}\right)$ mit $m / . .=262$ und $\mathrm{CMe}$ " mit $m / 2=57$

Wird das Isomerengemisch $\mathbf{2 a} / \mathbf{2 b}$ bei $-78^{\circ} \mathrm{C}$ ohne Zusatz von Triphenylphosphan mit elementarem Brom umgesetzt, beobachtet man zunächst eine Farbänderung von tiefrot nach braun. Erwärnt man anschließend auf Raumtemperatur, so setzt Gasentwicklung ein, und man erhält eine tiefgrüne Lösung sowie cinen hellgelhen Niederschlag. Nach Aufarbeitung des Filtrates isoliert man durch Kühlen auf

$20^{\circ} \mathrm{C}$ schwarzgüuc, nadelförmige Kristalle von Dibrom(1, 3-di-tert-butylcyclom pentadienyl)-5, $5^{\prime}-\left(\mathrm{I}^{\prime}, 3^{\prime}\right.$-di-tert-butyl-2',5'-cyclopentadieninickel 15$)$ die sich oberhalb $195^{\circ} \mathrm{C}$ zersetzen.

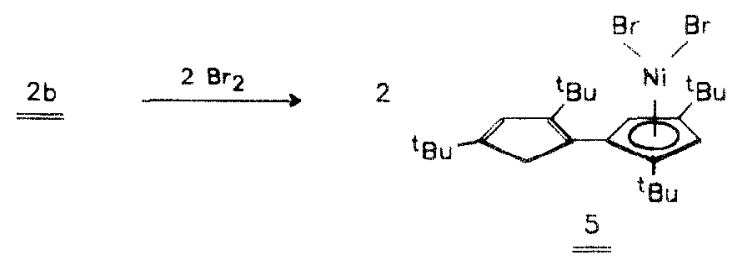

Schema 3. 


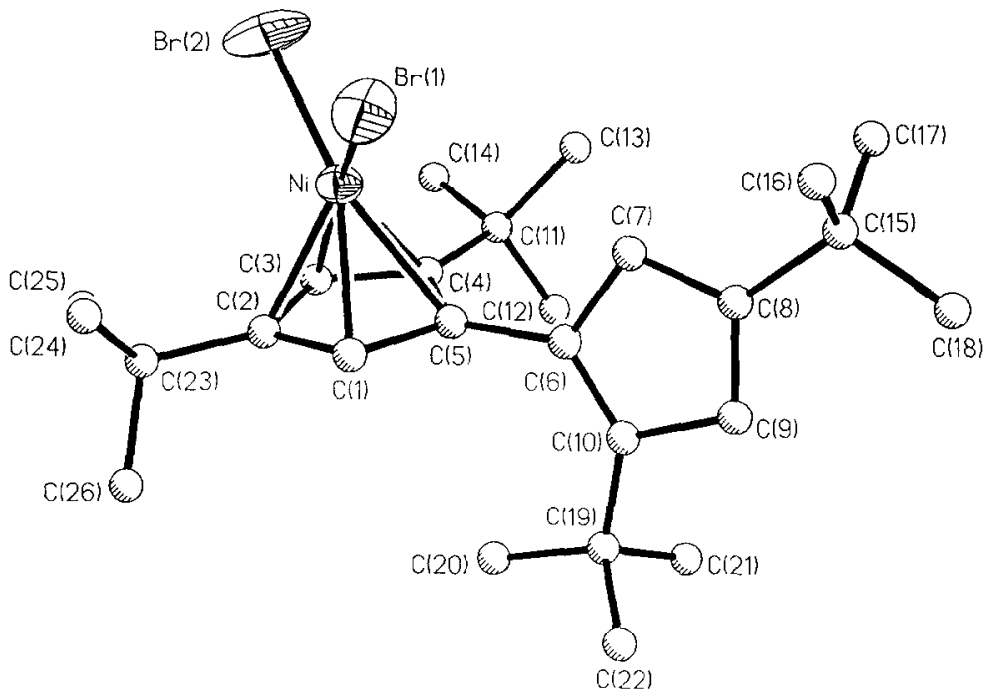

Fig. 1. Molekülstruktur von 5.

Verbindung 5 ist in Substanz luftstabil, in Diethylether tritt jedoch allmähliche Zersetzung ein. 5 ist stark paramagnetisch, wodurch ${ }^{1} \mathrm{H}$ - und ${ }^{13} \mathrm{C}$-NMRspektroskopische Untersuchungen nicht durchgeführt werden können. Das magnetische Moment von 5 beträgt $1.82 \mu \mathrm{B}$ und entspricht damit nahezu dem Idealwert von $1.73 \mu \mathrm{B}$ für das Vorhandensein eines ungepaarten Elektrons im Molekül.

Im ESR-Spektrum von $5(\nu=9.4160 \mathrm{GHz}$ ) beobachtet man bei einer Feldstärke von $317.48 \mathrm{mT}$ eine Absorption. Aus dem daraus berechneten $\mathrm{g}$-Wert von 2.1196 ergibt sich, daß dem Metall in 5 die Oxidationsstufe + III zukommt [7,15-18].

\section{Molekülstruktur von 5}

Die Röntgenstrukturanalyse [19*] von 5 zeigt ein Dibrom-di-tert-butyl(cyclopentadienyl)nickel-Fragment, das als weiteren Liganden am Cp-Ring eine Di-tertbutylcyclopentadienyl-Einheit trägt (Fig. 1). Die Ebenen der beiden $\mathrm{C}_{5}$-Ringe des Moleküls sind in bezug auf die zentrale $\mathrm{C}(5)-\mathrm{C}(6)$ Achse um einen Betrag von $78.3^{\circ}$ gegeneinander verdreht, so daß die tert-Butyl-Einheiten räumlich nahezu maximalen Abstand aufweisen (Fig. 2). Der C(5)-C(6)-Abstand entspricht mit einer Länge von $1.501(21) \AA$ einer Einfachbindung. Im koordinierten Teil des Liganden sind die C-C-Distanzen unterschiedlich; sie differieren zwischen $1.377(20) \AA[C(1)-C(5)]$ und 1.455(20) $\AA[C(4) C(5)]$. Der basale Fünfring ist zum Nickel nicht exakt pentahapto koordiniert. Die Abstände des Metalls zu den Kohlenstoff-Atomen des Cp-Gerüstes schwanken zwischen 2.055(12) $\AA$ [Ni-C(3)] und 2.148(14) $\AA$ [Ni-C(5)]. Der Abstand des Metallatoms zur Ringmitte beträgt $1.733 \AA$. Beide am Nickel koordinierten Brom-Atome sind zum Metall nahezu äquidistant; die Abstände liegen bei $2.286(3)$ und 2.290(2) $\AA$. Mit dem Metall bilden sie einen Winkel $(\mathrm{Br}-\mathrm{Ni}-\mathrm{Br})$ von $99.3(1)^{\circ}$.

In dem o-gebundenen Cyclopentadienyl-Liganden sind die RingkohlenstoffAtome $C(6) / C(10)$ sowie $C(8) / C(9)$ über Doppelbindungen verknüpft, mit Di- 


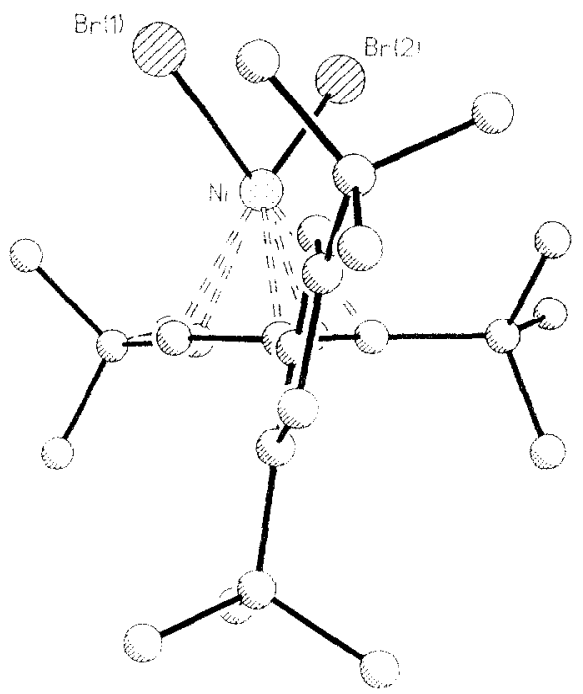

Fig. 2. Seitenansicht von 5.

stanzen von $1.344(22) \AA$ und $1.376(23) \AA$. Einfachbindungen werden zwischen den C-Atomen $\mathrm{C}(6) / \mathrm{C}(7)$. $\mathrm{C}(7) / \mathrm{C}(8)$ und $\mathrm{C}(9) / \mathrm{C}(10)$ gefunden. Diese Abstände variieren zwischen $1.486(21), 1.424(22)$ und $1.488(26) \AA$.

\section{Fazit}

Das Verhalten des Tetra-tert-butyl-dihydropentafulvalens in der $\pi$-Komplexchemie des Nickels ist äußerst überraschend. So liegt diese Verbindung sowohl als Neutralligand als auch in Form seines Mono- und Dianions vor. wobei $\eta^{\bar{*}}: \eta^{\bar{*}}$.

Tabelle 1

Bindungslängen (A) von 5

\begin{tabular}{|c|c|c|c|}
\hline $\mathrm{Br}(2)-\mathrm{Ni}$ & $2.286(3)$ & $\mathrm{Br}(\mathrm{l})-\mathrm{Ni}$ & $2.290(2)$ \\
\hline $\mathrm{Ni}-\mathrm{Cl}(1)$ & $2.069(13)$ & $\mathrm{Ni}-\mathrm{C}(2)$ & $2.107(14)$ \\
\hline $\mathrm{Ni}-\mathrm{C}(3)$ & $2.055(12)$ & $\mathrm{Ni} C(4)$ & $2.144(13)$ \\
\hline $\mathrm{Ni} C(5)$ & $2.148014 ?$ & $C(1) C(2)$ & $1.422(20)$ \\
\hline$C(1)-C(5)$ & $1.377(20)$ & $C(2) C(3)$ & $1.378(19)$ \\
\hline$C(2)-C(23)$ & $1.503(23)$ & $C(3)-C(4)$ & $1.392(20)$ \\
\hline$(4)-(5)$ & $1.455(20)$ & $(74)-(11)$ & $1.508(21)$ \\
\hline$C(5)-C(6)$ & $1.501(21)$ & $(16)-C(7)$ & 1.4862211 \\
\hline$C(6)-C(10)$ & $1.344(22)$ & $C(7)-C(8)$ & $1.424(22)$ \\
\hline$(8)-(9)$ & $1.376(23)$ & $C(8)-C(15)$ & $1.533(24)$ \\
\hline$C(9), C(10)$ & $1.488(26)$ & $C(10)-(119)$ & $1.515(23)$ \\
\hline$C(11)-C(12)$ & 1.5071221 & $(111)-(13)$ & 1.5900250 \\
\hline $\mathrm{C}(11) \cdots \mathrm{C}(14)$ & $1.547(23)$ & $C(15)-C(16)$ & 1.431129 \\
\hline$C(15)-C(17)$ & $1.451(27)$ & $((15)-C(18)$ & $1517(29)$ \\
\hline$C(19)-C(20)$ & $1.515(23)$ & $(19) \times(121)$ & $1.507(24)$ \\
\hline$C(19) C(22)$ & $1.472(22)$ & $C(23)-C(24)$ & $1.559(21)$ \\
\hline$C(23)-C(25)$ & $1.545(23)$ & $(723)-C(26)$ & $1.532020)$ \\
\hline
\end{tabular}


Tabelle 2

Bindungswinkel $\left({ }^{\circ}\right)$ von 5

\begin{tabular}{|c|c|c|c|}
\hline $\operatorname{Br}(2)-\mathrm{Ni}-\mathrm{Br}(1)$ & $99.3(1)$ & $\mathrm{Br}(2)-\mathrm{Ni}-\mathrm{C}(1)$ & $154.5(4)$ \\
\hline $\mathrm{Br}(1)-\mathrm{Ni}-\mathrm{C}(1)$ & $99.1(4)$ & $\mathrm{Br}(2)-\mathrm{Ni}-\mathrm{C}(2)$ & $114.7(4)$ \\
\hline $\operatorname{Br}(1)-\mathrm{Ni}-\mathrm{C}(2)$ & $126.7(4)$ & $\mathrm{C}(1)-\mathrm{Ni}-\mathrm{C}(2)$ & $39.8(6)$ \\
\hline $\operatorname{Br}(2)-\mathrm{Ni}-\mathrm{C}(3)$ & $94.7(4)$ & $\mathrm{Br}(1)-\mathrm{Ni}-\mathrm{C}(3)$ & $164.0(4)$ \\
\hline $\mathrm{C}(1)-\mathrm{Ni}-\mathrm{C}(3)$ & $65.2(5)$ & $\mathrm{C}(2)-\mathrm{Ni}-\mathrm{C}(3)$ & $38.7(5)$ \\
\hline $\mathrm{Br}(2)-\mathrm{Ni}-\mathrm{C}(4)$ & $108.7(4)$ & $\mathrm{Br}(1)-\mathrm{Ni}-\mathrm{C}(4)$ & $139.7(4)$ \\
\hline $\mathrm{C}(1)-\mathrm{Ni}-\mathrm{C}(4)$ & $66.1(5)$ & $\mathrm{C}(2)-\mathrm{Ni}-\mathrm{C}(4)$ & $65.9(5)$ \\
\hline $\mathrm{C}(3)-\mathrm{Ni}-\mathrm{C}(4)$ & $38.6(5)$ & $\mathrm{Br}(2)-\mathrm{Ni}-\mathrm{C}(5)$ & $147.6(4)$ \\
\hline $\mathrm{Br}(1)-\mathrm{Ni}-\mathrm{C}(5)$ & $105.7(4)$ & $\mathrm{C}(1)-\mathrm{Ni}-\mathrm{C}(5)$ & $38.1(6)$ \\
\hline $\mathrm{C}(2)-\mathrm{Ni}-\mathrm{C}(5)$ & $64.9(6)$ & $\mathrm{C}(3)-\mathrm{Ni}-\mathrm{C}(5)$ & $64.3(5)$ \\
\hline $\mathrm{C}(4)-\mathrm{Ni}-\mathrm{C}(5)$ & $39.6(5)$ & $\mathrm{Ni}-\mathrm{C}(1)-\mathrm{C}(2)$ & $71.5(8)$ \\
\hline $\mathrm{Ni}-\mathrm{C}(1)-\mathrm{C}(5)$ & $74.1(8)$ & $C(2)-C(1)-C(5)$ & $109.3(12)$ \\
\hline $\mathrm{Ni}-\mathrm{C}(2)-\mathrm{C}(1)$ & $68.6(7)$ & $\mathrm{Ni}-\mathrm{C}(2)-\mathrm{C}(3)$ & $68.6(8)$ \\
\hline$C(1)-C(2)-C(3)$ & $105.0(13)$ & $\mathrm{Ni}-\mathrm{C}(2)-\mathrm{C}(23)$ & $125.1(9)$ \\
\hline$C(1)-C(2)-C(23)$ & $125.5(12)$ & $C(3)-C(2)-C(23)$ & $129.4(13)$ \\
\hline $\mathrm{Ni}-\mathrm{C}(3)-\mathrm{C}(2)$ & $72,7(7)$ & $\mathrm{Ni}-\mathrm{C}(3)-\mathrm{C}(4)$ & $74.2(7)$ \\
\hline$C(2)-C(3)-C(4)$ & $113.2(13)$ & $\mathrm{Ni}-\mathrm{C}(4)-\mathrm{C}(3)$ & $67.2(7)$ \\
\hline $\mathrm{Ni}-\mathrm{C}(4)-\mathrm{C}(5)$ & $70.3(7)$ & $C(3)-C(4)-C(5)$ & $103.7(12)$ \\
\hline $\mathrm{Ni}-\mathrm{C}(4)-\mathrm{C}(11)$ & $126.9(10)$ & $C(3)-C(4)-C(11)$ & $125.8(13)$ \\
\hline$C(5)-C(4)-C(11)$ & $130.5(13)$ & $\mathrm{Ni}-\mathrm{C}(5)-\mathrm{C}(1)$ & $67.8(8)$ \\
\hline $\mathrm{Ni}-\mathrm{C}(5)-\mathrm{C}(4)$ & $70.0(8)$ & $C(1)-C(5)-C(4)$ & $108.5(13)$ \\
\hline $\mathrm{Ni}-\mathrm{C}(5)-\mathrm{C}(6)$ & $128.6(9)$ & $C(1)-C(5)-C(6)$ & $124.4(12)$ \\
\hline$C(4)-C(5)-C(6)$ & $127.1(13)$ & $C(5)-C(6)-C(7)$ & $122.2(12)$ \\
\hline$C(5)-C(6)-C(10)$ & $130.2(14)$ & $C(7)-C(6)-C(10)$ & $107.1(14)$ \\
\hline$C(6)-C(7)-C(8)$ & $107.2(13)$ & $C(7)-C(8)-C(9)$ & $108.9(14)$ \\
\hline$C(7)-C(8)-C(15)$ & $122.9(14)$ & $C(9)-C(8)-C(15)$ & $128.2(15)$ \\
\hline$C(8)-C(9)-C(10)$ & $107.2(15)$ & $C(6)-C(10)-C(9)$ & $109.4(14)$ \\
\hline$C(6)-C(10)-C(19)$ & $131.3(16)$ & $C(9)-C(10)-C(19)$ & $118.9(15)$ \\
\hline$C(4)-C(11)-C(12)$ & $110.1(12)$ & $C(4)-C(11) C(13)$ & $113.8(13)$ \\
\hline$C(12)-C(11)-C(13)$ & $108.3(14)$ & $C(4)-C(11)-C(14)$ & $109.9(13)$ \\
\hline$C(12)-C(11)-C(14)$ & $107.6(13)$ & $C(13)-C(11)-C(14)$ & $107.0(13)$ \\
\hline$C(8)-C(15)-C(16)$ & $113.0(17)$ & $C(8)-C(15)-C(17)$ & $110.8(15)$ \\
\hline$C(16)-C(15)-C(17)$ & $108.9(17)$ & $C(8)-C(15)-C(18)$ & $109.4(15)$ \\
\hline$C(16)-C(15)-C(18)$ & $106.7(17)$ & $C(17)-C(15)-C(18)$ & $107.8(17)$ \\
\hline$C(10)-C(19)-C(20)$ & $114.8(14)$ & $C(10)-C(19)-C(21)$ & $108.1(13)$ \\
\hline$C(20)-C(19)-C(21)$ & $107.5(13)$ & $C(10)-C(19)-C(22)$ & $108.7(13)$ \\
\hline$C(20)-C(19)-C(22)$ & $109.3(14)$ & $C(21)-C(19)-C(22)$ & $108.2(14)$ \\
\hline$C(2)-C(23)-C(24)$ & $111.6(12)$ & $C(2)-C(23)-C(25)$ & $109.4(13)$ \\
\hline$C(24)-C(23)-C(25)$ & $108.1(12)$ & $C(2)-C(23)-C(26)$ & $110.3(12)$ \\
\hline$C(24)-C(23)-C(26)$ & $108.9(12)$ & $C(25)-C(23)-C(26)$ & $108.4(12)$ \\
\hline
\end{tabular}

$\eta^{5}: \eta^{2}-, \eta^{2}: \eta^{2}$ - und $\eta^{5}: \eta^{0}$-Koordinationen beobachtet werden. Paramagnetische Nickel(I)- und Nickel(III)-Komplexe wie auch diamagnetische Nickel(II)-Komplexe können mit diesem Ligandensystem stabilisiert werden.

\section{Experimenteller Teil}

Sämtliche Arbeiten wurden unter Ausschluß von Luft und Feuchtigkeit unter Argon durchgeführt; verwendete Geräte, Chemikalien und Lösungsmittel waren entsprechend vorbereitet. Schmelzpunkte (n. korr.): Büchi 510 Schmelzpunktbestimmungsapparatur, Probenröhrchen abgeschmolzen. NMR (Bruker AM 300): 
${ }^{1}$ H-NMR $(300.1 \mathrm{MHz})$ ext. TMS, ${ }^{13} \mathrm{C}\left\{{ }^{1} \mathrm{H}\right\}-\mathrm{NMR}(75.4 \mathrm{MHz})$ ext. TMS. ${ }^{31} \mathrm{P}\left\{{ }^{1} \mathrm{H}\right\}-$ NMR (121.5 MHz) ext. (85proz. $\mathrm{H}_{3} \mathrm{PO}_{4}$ ). Massenspektren: Finnigan MAT $311 \mathrm{~A}$ (70 eV, $300 \mu \mathrm{A}$ Emission); es sind nur charakteristische Fragment-Ionen angegeben. CH-Analysen: Mikroanalytisches Laboratorium Beller, Göttingen: analytisches I abor der Fakultät für Chemie der Universität Bielefeld. FSR: Bruker FR 42019.42 GHz, $T=298 \mathrm{~K}$. Messung in Dichlormethan). Magnetische Messungen: Elektromagner Bruker BM-1. Mikrowage Sartorius 4411 120* I. IR-Spektrum: Mattson Polaris FTIR/Atari 1040 ST.

Dicarbonyll $1,1^{\prime}, 3,3^{\prime}$-tetra-ter buty $1,5,5^{\prime}$-pentafullalen $)\left(1,7^{\prime}, 3,3^{\prime}\right.$-tetra-tert-buty $/ .5,5^{\prime}$

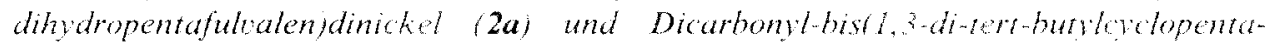
dienyl)-5,5'-1/'.3'-di-tert-butplcyclopentadien)dinickel (2b)

$\mathrm{Zu}$ einer auf $-30^{\circ} \mathrm{C}$ gekuhlten Lösung von $2.36 \mathrm{~g}(6.60 \mathrm{mmol}) 1$ in $30 \mathrm{ml} \mathrm{THF}$ tropft man eine Lösung von $6.60 \mathrm{mmol}$ Methyllithium in Diethylether und erwärmt auf Raumtemperatur. Man rihht $12 \mathrm{~h}$, versezt mit $300 \mathrm{~g}(17.6 \mathrm{mmol}) \mathrm{Ni}\left(\mathrm{CO}_{4}\right.$ in $30 \mathrm{ml}$ THF und erhitzt das braun-schwarze Gemisch 3 h unter Rückfluf. Das Solvens wird i. Vak. abdestllient der Rückstand in $50 \mathrm{ml}$ Petrolether aufgenommen und zentrifugiert. Die überstehende tiefrote Lösung wird eingeengt und an $\mathrm{Al}_{3} \mathrm{O}_{3}$ (Akt. III) Petrolether chromatographiert. Die zweite, kirschrote Fraktion wird aufgefangen und eingeengt, Man isoliert ein rotes amomhes Pulver. Ausbeute: $0.92 \mathrm{~g}(32 \%)$. Schmp : $229 \ldots 311^{\circ} \mathrm{C}$.

'H-NMR $\left(C_{6} D_{6}\right): 2 a d=1.21,1.27,1.32 .1 .36\left(4 \mathrm{~s},{ }^{2} B u\right), 3,10,4.84,5.13,6.21 .6 .36$ (5s, $\mathrm{Cp}-\mathrm{H}) ; 2 \mathbf{b} \delta=1.16,125,1.35,1.39\left(4 \mathrm{~s},{ }^{\mathrm{t}} \mathrm{Bu}\right), 3.10,4.96,5.08,5.11 .6 .25(5 \mathrm{~s}$ (p-H). ${ }^{1} \mathrm{C}-\mathrm{NMR}\left(\mathrm{C}_{6} \mathrm{D}_{6}\right): \delta=30.68,31.88,32.44,32.29(\mathrm{CMc}), 31.04 .32 .53$. 33.04, 34.20( $\left(\mathrm{CMe}_{3}\right), 41.98 .51 .08,91.35,91.58,91.71,97.30,97.67,99.06,109.00$. $119.42,119.59,120.23,120,82,121.01,131.81,134.82,13508,150.96,152.03,157.01$

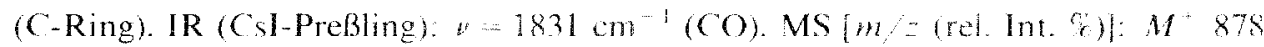
(32.7). $M^{+}-2 \mathrm{CO} 822(4.1), M^{\prime}-2 \mathrm{CO}-{ }^{\mathrm{B}} \mathrm{Bu} 765(18.8), M^{+}-2 \mathrm{CO}-2^{\mathrm{t}} \mathrm{Bu} 708$ (46.4), $\left.{ }^{1} \mathrm{Bu}_{4} \mathrm{C}_{10} \mathrm{H}_{5} \mathrm{NiCO} 4.39(22.6) .{ }^{1} \mathrm{Bu}_{4} \mathrm{C}_{10} \mathrm{H}_{5} \mathrm{Ni}^{+} 41\right](100.0) . \mathrm{Ce}^{2}: 57(15.4)$ Magn. Suszept. $[\mu \mathrm{B}]: \mu_{\text {Merf }}-2.58$.

Gef. C, 73.64; H. 9.13. C ${ }_{4} \mathrm{H}_{42} \mathrm{Ni}_{2} \mathrm{O},(880.6)$ : Ber. C. 73.65; H. 9.389.

Dibrom-bis [triphenylphosphan](1,1',3,3'-tetra-tert-buty l-5,5' pentafultalendinickel (3)

$\mathrm{Zu}$ einer Lösung von $0.56 \mathrm{~g}(0.60 \mathrm{mmol}) 2 \mathrm{a}$ in $30 \mathrm{~m}$ Diethylether troplt man bei $-40^{\circ} \mathrm{C} 0.14 \mathrm{~g}(0.88 \mathrm{mmol})$ Brom in $20 \mathrm{ml}$ Diethylether und rihrt $1 \mathrm{~h}$. Anschliekend werden $0.35 \mathrm{~g}(1.34 \mathrm{mmol})$ Triphenylphosphan. gelöst in $20 \mathrm{~m}$ ! Toluol, zugegeben. Man erwärmt auf Raumtemperatur, filtriert, engt $i$. Vak ein und kihlt auf $-60^{\circ} \mathrm{C}$ 3 wird in Form dunkelroter Kristalle erhalten. Ausbeute 0.10 y $99 \%$ Schmp. $269^{\circ} \mathrm{C}$

'H-NMR $\left(C_{6} D_{6}\right): \delta=0.97,1.11,1.48,1.27(4 \mathrm{~s}, 4 \times 9$ H. $4 \times$ Bu). 2.94. 3.03, 5.54. $\left.6.67(4 \mathrm{~m} .4 \times \mathrm{Cp}-\mathrm{H}) .7 .20 \cdots 7.30(\mathrm{~m}, 30 \mathrm{Ph}-\mathrm{H}) .{ }^{31} \mathrm{P}-\mathrm{NMR}\left(\mathrm{C}_{0} \mathrm{D},\right)_{0}\right) 8-28.40$.

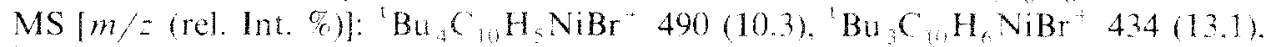
${ }^{1} \mathrm{Bu}_{4} \mathrm{C}_{10} \mathrm{H}_{5} \mathrm{Ni}$ '411(1.4), $\left.\mathrm{Bu}_{2} \mathrm{C}_{10} \mathrm{H}_{7} \mathrm{NiBr}^{+} 378(1.4), \mathrm{P}_{6} \mathrm{CH}_{5}\right) \quad 262(61.9) \mathrm{CM}$ $57(100.0)$.

Gef. C. 64.42: H. 7.03. $\mathrm{C}_{2} \mathrm{H}_{-4} \mathrm{Br}_{2} \mathrm{Ni}_{2} \mathrm{P}$ (1153.4): Ber. C.64.56: H. 607\%

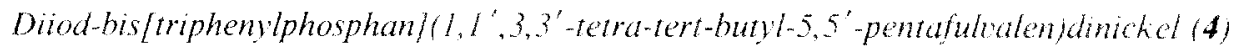

$\mathrm{Zu}$ einer Lösung von $1.00 \mathrm{~g}(1.15 \mathrm{mmol}) \mathbf{2 a}$ in $50 \mathrm{ml}$ Diethylether tropft man bei $-35^{\circ} \mathrm{C}$ eine lösung von $0.40 \mathrm{~g}(1.57 \mathrm{mmol})$ Iod in $10 \mathrm{ml}$ Diethyther und rühr 
$1.5 \mathrm{~h}$. Anschließend kühlt man auf $-50^{\circ} \mathrm{C}$ und versetzt mit $0.82 \mathrm{~g}(3.13 \mathrm{mmol})$ Triphenylphosphan in $20 \mathrm{ml}$ Toluol. Man erwärmt auf Raumtemperatur, rührt weitere $12 \mathrm{~h}$ und entfernt die Solventien i. Vak.. Der Rückstand wird in Toluol/ Diethylether $(1: 1)$ aufgenommen und filtriert. Man engt $\mathrm{i}$. Vak. ein und nimmt in Petrolether auf. Kühlen auf $-20^{\circ} \mathrm{C}$ liefert rote Kuben. Ausbeute: $0.10 \mathrm{~g}(14 \%)$, Schmp.: $234-236^{\circ} \mathrm{C}$.

${ }^{1} \mathrm{H}-\mathrm{NMR}\left(\mathrm{C}_{6} \mathrm{D}_{6}\right): \delta=0.88,1.06,1.43,1.67\left(4 \mathrm{~s}, 4 \times 9 \mathrm{H} 4 \times{ }^{\mathrm{t}} \mathrm{Bu}\right), 2.86,2.95,3.10$, $5.45(4 \mathrm{~m}, 4 \times \mathrm{Cp}-\mathrm{H}), 7.03(\mathrm{~m}, 30 \mathrm{Ph}-\mathrm{H}) .{ }^{13} \mathrm{C}-\mathrm{NMR}\left(\mathrm{C}_{6} \mathrm{D}_{6}\right): \delta=30.53,31.56$, 31.75, $32.08\left(\mathrm{CMe}_{3}\right), 32.54,33.12,33.85,34.28\left(\mathrm{CMe}_{3}\right), 85.56,86.17,90.28,90.83$, 111.16, 117.97, 124.58, 128.78, 129.01, 131.61 (C-Ring), 132.04, 133.95, 134.15 (C-Phenyl). ${ }^{31} \mathrm{P}-\mathrm{NMR}\left(\mathrm{C}_{6} \mathrm{D}_{6}\right): \delta=36.80$. MS $\left[\mathrm{m} / z\right.$ (rel. Int. $\%$ )]: ${ }^{1} \mathrm{Bu}_{4} \mathrm{C}_{10} \mathrm{H}_{5} \mathrm{NiI}^{+}$ 538 (26.3), ${ }^{\mathrm{t}} \mathrm{Bu}_{3} \mathrm{C}_{10} \mathrm{H}_{6} \mathrm{NiI}^{+} 482$ (20.4), ${ }^{\mathrm{t}} \mathrm{Bu}_{2} \mathrm{C}_{10} \mathrm{H}_{7} \mathrm{NiI}^{+} 426(8.8),{ }^{\mathrm{B}} \mathrm{Bu}_{4} \mathrm{C}_{10} \mathrm{H}_{5} \mathrm{Ni}^{+}$ 411 (1.6), $\mathrm{P}\left(\mathrm{C}_{6} \mathrm{H}_{5}\right)_{3}{ }^{+} 262(100.0)$.

Gef. C, 59.03; H, 5.84. $\mathrm{C}_{62} \mathrm{H}_{70} \mathrm{I}_{2} \mathrm{Ni}_{2} \mathrm{P}_{2}$ (1248.4): Ber. C, 59.65; H, 5.65\%.

Dibrom(1,3-di-tert-butylcyclopentadienyl)-5,5'-(1',3'-di-tert-butyl-2',5'-cyclopentadien)nickel (5)

$\mathrm{Zu}$ einer Lösung von $0.70 \mathrm{~g}(0.80 \mathrm{mmol}) \mathbf{2 b}$ in $50 \mathrm{ml}$ Diethylether tropft man bei $-78^{\circ} \mathrm{C}$ eine Lösung von $0.26 \mathrm{~g}$ (1.63 $\left.\mathrm{mmol}\right)$ Brom in $10 \mathrm{ml}$ Diethylether, wobei sich die Reaktionsmischung schwarz-braun färbt. Anschließend erwärmt man auf Raumtemperatur, rührt weitere $12 \mathrm{~h}$ und erhält eine tiefgrüne Lösung. Diese wird eingeengt, der Rückstand in Diethylether aufgenommen und filtriert. Die Lösung wird auf $-20^{\circ} \mathrm{C}$ gekühlt, wobei 5 in Form schwarz-grüner Kristalle erhalten wird. Ausbeute: $0.29 \mathrm{~g} \mathrm{(46 \% ),} \mathrm{Schmp.:} 195^{\circ} \mathrm{C}$ (Zers.).

MS $\left[m / z\right.$ (rel. Int. \%)]: $M^{+}-\mathrm{Br} 490$ (4.5), ${ }^{\mathrm{t}} \mathrm{Bu}_{3} \mathrm{C}_{10} \mathrm{H}_{6} \mathrm{NiBr}^{+} 434$ (6.2), ${ }^{\mathrm{t}} \mathrm{Bu}_{4} \mathrm{C}_{10} \mathrm{H}_{5} \mathrm{Ni}^{+} 411$ (3.9), ${ }^{\mathrm{t}} \mathrm{Bu}_{3} \mathrm{C}_{10} \mathrm{H}_{4} \mathrm{Ni}^{+} 353$ (8.4), ${ }^{\mathrm{t}} \mathrm{Bu}_{3} \mathrm{C}_{10} \mathrm{H}_{6}{ }^{+} 297$ (16.5), $\mathrm{CMe}_{3}{ }^{+}$ 57 (100.0). ESR: $g=2.1196$. Magnet. Suszept. $[\mu \mathrm{B}]: \mu_{\mathrm{M}(\mathrm{eff})}=1.82$.

Gef. C, 53.38; H, 6.74. $\mathrm{C}_{26} \mathrm{H}_{41} \mathrm{Br}_{2} \mathrm{Ni}(572.1)$ : Ber. C, 54.78; H, 6.90\%.

\section{Dank}

Wir danken der Deutschen Forschungsgemeinschaft und dem Fonds der Chemischen Industrie für die gewährte finanzielle Unterstützung.

Frau U. Stuphorn, Fakultät für Chemie der Universität Bielefeld (Arbeitskreis Prof. Dr. A. Müller), danken wir für die magnetischen Messungen. Herrn Dr. H.-P. Korth, Universität-GH-Essen (Arbeitskreis Prof. Dr. R. Sustmann), gilt unser Dank für die Aufnahme des ESR-Spektrums.

\section{Literatur und Bemerkungen}

1 K.W. Barnett, J. Organomet. Chem., 78 (1974) 139.

2 H. Lehmkuhl, F. Danowski, R. Benn, R. Mynott und G. Schroth, Chem. Ber,, 119 (1986) 2542.

3 R. Brand, H.-P. Krimmer, H.-J. Lindner, V. Sturm und K. Hafner, Tetrahedron Lett., 23 (1982) 5131.

4 P. Jutzi und J. Schnittger, Chem. Ber., 122 (1989) 629.

5 P. Jutzi, J. Schnittger, J. Dahlhaus, D. Gestmann und H.-C. Leue, J. Organomet. Chem., 415 (1991) 117.

6 P. Jutzi, J. Schnittger und M.B. Hursthouse, Chem. Ber., im Druck.

7 M. Huhn, W. Kläui, L. Ramacher, R. Herbst-Irmer und E. Egert, J. Organomet. Chem., 398 (1990) 339. 
8 E.O. Fischer und C. Palm, Chem. Ber., 91 (1958) 1725.

9 T. Mise und H. Yamazaki, J. Organomet. Chem., 164 (1979) 391.

Io P. Eilbracht, U. Mayser und G. Tiedtke. Chem. Ber. 113 (1980) 1420

11 P. Eilbracht. Chem. Ber., $109(1976) 3136$.

12 L. R. Byers und L.F. Dahl, Inore. Chem.. 19 (1980) 680.

13 Durch die Deprotonierung won 1 mit Methyllithium liegt ein Gemisch aus dem Dianion und nicht metalliertem Liganden 1 vor, welche iber dit monoprotonierte Spezies in Gleichgewich skhen: Tetracarbonylnickel reagiert dann mit dem Dianion und 1 zu 2 a und mil den Monoanion a $2 \mathrm{~b}$. Die Entstehung von $\mathbf{2 a} / \mathbf{2 b}$ auf diese Weise ist unibersichthch. Eine verglechbarc Reaktion wn $\mathbf{C}$. Ve l. mit $\mathrm{Ni}(\mathrm{CO})_{4} z u\left[\mathrm{C}_{5} \mathrm{Me}_{5} \mathrm{Ni}(\mathrm{CO})\right]_{2}$ ist literaturbekannt $[9 !$.

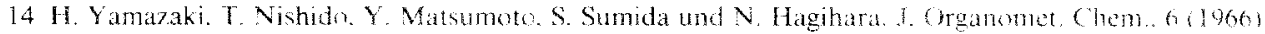
86.

15 Landolt-Bönstein, Zahtenwerk und Funktionen aus Physik. Chemie, Astronomie. Geophysik, Technik (J. Bartels. H. Borchers, H. Hansen. K.H. Hellwege, K. Schäfer und E. Schmidt. Hrag.) 0 Auflage. 11. Bd. 9. Teil. Springer Verlag. Berlin. 1962. S4-1.

16 S. Geschwind und J.P. Rameika, Appl. Phys. Supl. $33(1962) 370$.

17 J.C. Smart und B.L. Pinski, J. Am. Chem. Soc. 99 (1977) 956.

18 R.E Dessy. F.F. Stary. R.B. King und M. Waldrop. J. Am. Chem. Sod. 88 (1966) 471.

19 Daten zur Strukturanalyse von 5 (Standardabweichungen sind in Klammern angegeben). Summenformel $\mathrm{C}_{20} \mathrm{H}_{41} \mathrm{Br}_{2} \mathrm{Ni}$ : Molmase $572.1 \mathrm{~g} / \mathrm{mol}$ : Gitterkonstanten a $=14.8734, \mathrm{~A}, \mathrm{i}=15026(4) \mathrm{A}$ $\because=24.751(6)$ A. Zelwolumen $5571\left(2, \mathrm{~A}^{3}, Z=8 ; d_{\text {her }}=1.374 \mathrm{~g} / \mathrm{cm}^{3}\right.$ : Lincarer Aborptionskueffizient $3.576 \mathrm{~mm}$ 1; $F(000)=2360$. Kristallsystem orthorhombisch. Raumgruppe Phat MeBgeriat.

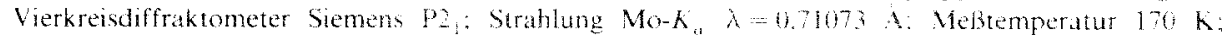
Melbereich $3 \leqslant 2 \theta \leqslant 50^{\circ} ; h, k, /$ Genzen $0 \rightarrow 17,0 \rightarrow 17,0 \rightarrow 29:$ Ahsomptionkorrekur mumensch: Zahl der gemessenen Reflexe 5460 . davon unabhänge 1899 mit $/ 2.20 /$ / s srakturauklärung mit

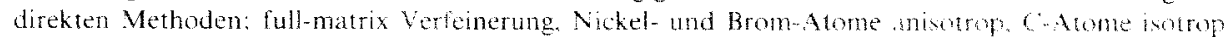

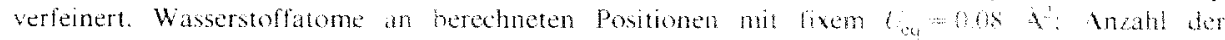

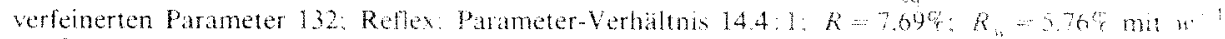
$=\sigma^{2}(F)$. Weltere Einzelheiten zur Kristallstruktur won 5 komen hem Fathinformationsentrum

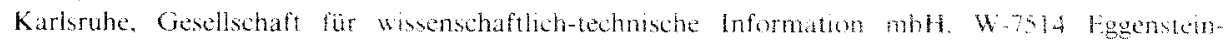
Leopoldshafen 2, unter Angabe der Hinterlegungsnummer CSD-5534, hor Aunonentamen und des: Zeilschriftenzitats angefordert werden.

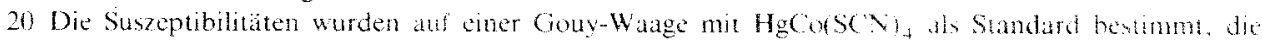
Korrektur für den Diamagnetismes des Systems berechneten wir neh der Inkremenomethol: nach Haberdity 